\title{
ÉTICA DEL DESARROLLO, DEMOCRACIA DELIBERATIVA Y CIUDADANÍA BIOLÓGICA. UNA ARTICULACIÓN EN CLAVE BIOPOLÍTICA AFIRMATIVA ${ }^{1}$
}

\author{
Raúl Villarroel \\ Universidad de Chile \\ rvillarr@uchile.cl
}

\begin{abstract}
Resumen / Abstract
El presente artículo indaga en los alcances teóricos y el impacto de las diversas concepciones del fenómeno del desarrollo. Atiende, en primer lugar, a la reflexión acerca de los fines y los medios del desarrollo que ha sido denominada "Ética del Desarrollo". Estima que el examen de esta visión es necesario establecerlo en concomitancia con la reflexión sobre la democracia participativa (deliberativa). El trabajo teórico que se ha venido haciendo con miras a la ampliación de la noción tradicional y restringida de "ciudadanía" política, le otorga un importante elemento de juicio para el examen de la democracia. Le permite entender de mejor manera el modo como se podría pensar en una confluencia de desarrollo y democracia participativa (deliberativa), mediando un ejercicio activo de la ciudadanía, entendida en un sentido ampliado, una de cuyas expresiones se encontraría en el concepto de "Ciudadanía biológica", que examina desde un enfoque de biopolítica afirmativa.

Palabras clave: ética del desarrollo, democracia deliberativa, ciudadanía, ciudadanía biológica, biopolítica afirmativa.
\end{abstract}

\section{ETHICS OF DEVELOPMENT, DELIBERATIVE DEMOCRACY AND BIOLOGICAL CITIZENSHIP}

This article will seek to inquire into the theoretical scope and consequences and impact that the conceptions of development have had. This will be, firstly, according to a certain kind of reflection about the purposes and the means of development so called "Development Ethics". This approach must be established in order to "Deliberative Democracy". Theoretical work that has been done, also during the last decades, to improve and expand the traditional and restricted notion of "political citizenship"-in the sense of a "Biological Citizenship", according to an affirmative biopolitical concept-, gives a relevant element of judgement that makes it possible to understand in a better way how it could be conceived the confluence of Development and Deliberative Democracy.

KEY WORDS: Development ethics, Deliberative Democracy, Citizenship, Biological Citizenship, Affirmative Biopolitics.

Este artículo es parte de la investigación asociada al Proyecto Fondecyt No 1120141 "La articulación entre éticas del desarrollo y teorías democráticas participativas (deliberativas). Una investigación-reflexión", cuyo investigador responsable es el autor. 


\section{Introducción}

RA El presente artículo ${ }^{2}$ buscará indagar en los alcances teóricos y las consecuencias e impacto que las diversas concepciones del desarrollo han tenido. Ello se hará, en primer lugar, atendiendo a la reflexión acerca de los fines y los medios del desarrollo que ha sido denominada "Ética del Desarrollo". Se estima que el examen de esta visión es necesario establecerlo en concomitancia con la reflexión sobre la "democracia participativa (deliberativa)". El trabajo teórico que se ha venido haciendo, también durante las últimas décadas, con miras a la ampliación de la noción tradicional y restringida de "ciudadanía" política, otorga un importante elemento de juicio, en tanto permite situar en un contexto de pertenencias, participación y reconocimientos al examen de la democracia. Ello hace posible entender de mejor manera el modo como se podría pensar en una confluencia de desarrollo y democracia participativa (deliberativa), mediando el ejercicio activo de la ciudadanía, entendida en un sentido ampliado, una de cuyas expresiones se encontraría en el concepto de "Ciudadanía biológica" que aquí se examina.

\section{La Ética del Desarrollo}

El giro aplicado de la filosofía (Cortina 2002), es la expresión variada y multidisciplinaria de la ética filosófica que consiste en el análisis de temas morales específicamente controversiales, que destacan por ocupar la atención de la sociedad civil, con especial énfasis en nuestra época (Cortina 2002b). Entre las variadas expresiones de ética aplicada existentes en la actualidad y a la cual se consagran destacados pensadores de nuestro tiempo, se puede mencionar aquella visión cuestionadora del paradigma socioeconómico vigente actualmente en el mundo, a la que se ha dado en denominar "Ética del Desarrollo", que constituye un referente teórico fundamental y se perfila como uno de los ejes problemáticos centrales de esta reflexión.

La Ética del Desarrollo es una reflexión crítica sobre los fines y medios que definen a los cambios sociales, económicos y culturales en los países y regiones pobres y en vías de desarrollo (Crocker 2002). Desde esta perspectiva, resulta posible enfatizar el rol activo que le cabe jugar a la dimensión ético-moral de la teoría y la práctica del desarrollo, paralelamente a sus componentes económicos, políticos y tecnocientíficos (Crocker 2002, 2008). Hoy se estima que no es suficiente una visión exclusivamente economicista del desarrollo, así como tampoco lo sería una idea que promueva el progreso social sacrificando los logros económicos. Ciertamente, el desarrollo -el progreso económico y social- depende de una gran variedad de factores, tales como la

2 Partes de este artículo (específicamente algunos pasajes de la pág. 280), se desprenden de reflexiones de Jorge Vergara Estévez, coinvestigador del Proyecto. 
tecnología, la iniciativa privada, las habilidades, el liderazgo, las políticas comerciales, la eficiencia de los sistemas fiscales, la seguridad social, las políticas públicas y hasta la ética empresarial (Conill 2006). Por lo mismo -como, por ejemplo, piensa Amartya Sen-, el desarrollo no puede ser reducido solo al crecimiento del Producto Interno Bruto o al aumento de algún indicador de la renta nacional (Sen 2000). En tal sentido, Sen afirma que el desarrollo no puede ser medido sin tener en cuenta el estilo de vida que pueden llevar las personas y sus libertades reales. Dicho con otras palabras, el concepto de desarrollo no puede limitarse al crecimiento de objetos inanimados de conveniencia, como incrementos del PNB (o del ingreso personal), o la industrialización, o el progreso tecnológico, o la modernización social (Sen 2002).

Según lo planteado por Denis Goulet, en su ya clásica obra Ética del Desarrollo. Guía teórica y práctica (1999), el estudio sistemático y deliberado de la Ética del Desarrollo se vincularía directamente al nacimiento de IDEA (International Development Ethics Association), ocurrido en el año 1987, en San José de Costa Rica (Goulet 1999, p. 25). La visión compartida por todos sus miembros respecto de la tarea que correspondía asumir a la ética establecía la necesidad de "diagnosticar problemas vitales con los que se enfrentan las sociedades humanas, orientar las decisiones políticas y clarificar las cuestiones de valor que envuelven esos problemas y esas políticas" (ibíd., p. 26). A partir de este acuerdo, entonces, según lo señala Goulet: "la ética del desarrollo adquirió un reconocimiento formal como campo interdisciplinario en los estudios sobre el desarrollo y la filosofía" (ibíd.).

En todo caso, es posible pensar que precedentes de esta Ética del Desarrollo se encontrarían ya en los debates y documentos que dieron lugar a la Declaración Universal de los Derechos Humanos de 1948 y en la misma declaración como tal. "En ella encontramos los elementos clave de lo que, todavía hoy, entendemos por «auténtico desarrollo» frente a posibles sucedáneos: se trata de aquella situación social en la que toda persona encuentra garantizados sus derechos básicos y dispone realmente de posibilidades para ejercer sus obligaciones y para llevar adelante proyectos de vida buena en armonía con su comunidad y con el medio ambiente" (Martínez Navarro 2007).

Considerando que, incluso en los países ricos, la pobreza y las desigualdades se han incrementado durante el último siglo, los esfuerzos para comprender y reducir estos flagelos sociales han adoptado diversas formas. La reflexión moral acerca de los fines y medios del desarrollo -donde "desarrollo" significa genéricamente beneficio social-, es uno de los esfuerzos importantes al respecto. Tal reflexión moral, que incluye la evaluación crítica del presente y la visualización de mejores expectativas de futuro, crecientemente, ha sido llamada también "Ética internacional del desarrollo", o "Ética del Desarrollo Global” (Crocker 2008). De este modo, la Ética del Desarrollo se propone contribuir a ampliar la discusión y la reflexión acerca del proceso de cambio social vinculado al desarrollo (Sen 2000), para orientar la formulación de estrategias, políticas y planes de acción, tendiendo a otorgar relevancia a factores normalmente desatendidos en las conceptualizaciones corrientes de las teorías respectivas.

David Crocker describe al menos cuatro fuentes originarias de esta modalidad de evaluación moral de la teoría y la práctica del desarrollo (Crocker 2008). En primer 
lugar, el trabajo de ciertos activistas y críticos sociales como, por ejemplo, Mohandas Gandhi en la India, Raúl Prebisch en América Latina y Frantz Fanon en África, que a comienzos de los años 40 del siglo anterior enjuiciaron al colonialismo y a la noción ortodoxa del desarrollo. Luego, en segundo término, estarían los planteamientos del pensador estadounidense Denis Goulet, influido por el economista Louis-Joseph Lebret y algunos cientistas sociales tales como, por ejemplo, Gunnar Myrdal, quienes argumentaron que el desarrollo requiere ser redefinido, desmitificado y lanzado a la arena del debate moral. Como una tercera fuente para la ética del desarrollo se podría considerar-según Crocker- el esfuerzo desplegado a comienzos de los años 70 por los filósofos angloamericanos para profundizar y ampliar el debate filosófico acerca de la superación del hambre y la ayuda alimenticia. Y por último, en cuarto lugar, estarían los trabajos de Paul Streeten y de Amartya Sen, economistas que han abordado las causas de la desigualdad económica global, el hambre y el subdesarrollo y han vinculado estos problemas, entre otras cosas, con una concepción del desarrollo explícitamente basada en principios éticos (ibíd.).

En general, este enfoque ético del desarrollo busca contravenir aquella otra visión que a partir de la segunda mitad del siglo XX se impuso en el mundo y que miraba al desarrollo como una cuestión de naturaleza eminentemente técnica, orientada a la planificación de los recursos materiales, mediante acciones de ingeniería social y con el propósito de introducir a las naciones en un camino de crecimiento económico sostenido. No tenemos aquí la oportunidad ni el espacio para establecer la genealogía exacta de este enfoque. Baste con recordar que ellas estuvieron asociadas, principalmente, en las décadas de los años 50 y 60 al énfasis puesto por los economistas y planificadores en la formación de capital y la transferencia de tecnología moderna en vistas de una creciente industrialización, cuya expresión posterior en ámbitos agrícolas, poblacionales y laborales evidenció su interés expansivo. De hecho, se puede decir que se trató de un impulso que tuvo su origen en la reconstrucción de Europa tras la Segunda Guerra Mundial (Plan Marshall), pero que muy pronto desvió su atención hacia aquellas regiones del mundo consideradas pobres y excluidas (Latinoamérica, Asia y África), en la perspectiva de integrarlas a este mismo movimiento reconstructivo.

Aunque discutido en su momento, el take off del economista liberal estadounidense Walter Rostow parece ser el epítome expresivo de las bondades asociadas a esta concepción del desarrollo esperanzada en la posibilidad de remontar definitivamente su ausencia y las dificultades, las resistencias y los obstáculos asociados a la superación de la pobreza. Como pensaba Rostow, es necesario hacer avanzar a las sociedades en estado de transición hacia el "despegue económico" (take off), de tal manera que puedan explotar los frutos de la ciencia moderna y puedan vencer sus rendimientos decrecientes (Rostow 1960). Así, también, podrían superar el "atraso" en el que habían vivido y ajustar sus estructuras sociales y sus economías al proceso de "modernización".

En relación con ello, se puede sostener que esta particular ortodoxia "desarrollista" prescindió absolutamente de consideraciones éticas y no parece haber tenido preocupación alguna por las personas sobre las cuales se buscó implementar. Excepto, claro está, de una preocupación pragmática e ideológica, que concernía a la posibilidad de hacerlas complacientes y obedientes a sus propios fines, sobreentendiendo que sus metas 
constituían algo digno de ser admitido y diseminado vertiginosamente en la mayor medida de lo posible. "Millares de economistas, administradores e ingenieros fueron emergiendo de los ámbitos profesionales para convertirse en arquitectos sociales de un mundo nuevo, que actuaban como expertas comadronas que querían transferir a las naciones «subdesarrolladas» las instituciones, las prácticas y la tecnología de los países industrializados [...] Con arrogante autosuficiencia, emprendieron la tarea de dar nueva forma a cualquier cultura o sistema de valores", afirma el economista estadounidense Denis Goulet en su afamada obra Ética del Desarrollo. Guía teórica y práctica (Goulet 1999, p. 27).

No obstante, tras ya varias décadas de implementación de esta concepción doctrinaria de "desarrollo", considerado hasta ahora objetivo primordial de la política económica de las naciones y estrategia conjunta internacional, los eticistas del desarrollo piensan que parece haber llegado la hora de llevarlo a la arena del debate filosófico y moral. Creen que es necesario que comparezca públicamente y responda a las grandes interrogantes que conciernen a sus fines, es decir, responda respecto del real crecimiento económico que puede propiciar o que puede inhibir; de la satisfacción o insatisfacción de necesidades básicas que es capaz de producir; de las supervivencias culturales que puede permitir o que puede impedir a través de su despliegue; de los traspasos de poder que facilita o interfiere entre las clases sociales y otras materias semejantes que constituyen asuntos generadores de profundos y -hasta este minuto-irresolubles conflictos de valor. Todo ello ha llevado al descrédito a dicha doctrina desarrollista.

Los filósofos del desarrollo, y otros eticistas involucrados en el tema intentan proponer parámetros basados éticamente para repensar el desarrollo; desde perspectivas interdisciplinarias, y no solo comprendiendo su objeto de estudio como aquel cambio social deseable, sino también argumentando y promoviendo concepciones específicas sobre dicho cambio. De tal manera, intentan formular aquellos principios éticos que consideran decisivos para el cambio social, analizando y evaluando las dimensiones morales de las teorías vigentes, en busca de criterios que favorezcan una mejor comprensión de las dificultades suscitadas a partir de la implementación de las políticas y las prácticas efectivas de desarrollo (Crocker 2008).

No obstante, debemos entender que todo ello requiere de una perspectiva de democracia concebida en términos de discusión pública y participación ciudadana. Es decir, atendida la idea de que una ética del desarrollo promueve una dinámica socioeconómica fundada en la agencia de los propios individuos y en la posibilidad efectiva que a ellos se les presente de vivir la vida que estimen más valiosa, parece evidente que solo una estructura política participativa podría hacer viable una idea de desarrollo así concebida. Se asume, al mismo tiempo, que un enfoque ético del desarrollo, interpretado en la clave de las capacidades humanas (Sen 2000), no piensa en unos “ciudadanos que se 'desentienden' de tomar ellos mismos decisiones políticas y no quieren participar de manera activa en la ciudadanía, sino que prefieren delegar esa responsabilidad a gente especializada en tomar esas decisiones, y que de alguna manera los representen" (Peñas, P. 2011, p. 281). Un desarrollo orientado éticamente se funda, entonces, en una "Democracia participativa (deliberativa)", tal como ha sido ampliamente tematizada por Habermas, Macpherson, Held, Rawls, Nino, Elster y otros 
autores. En consecuencia, la reflexión analítica y crítica acerca de la naturaleza, los méritos, los desafíos y los límites de una democracia participativa (deliberativa), se constituyen en una importante posibilidad para favorecer el despliegue de las capacidades y el poder de decisión sobre su propio destino que tienen los individuos en el contexto social (Crocker 2005).

\section{Las concepciones participativas (deliberativas) de la democracia}

Es un asunto convencional diferenciar entre dos modelos de democracia, una democracia liberal o representativa, y una democracia participativa. En esta última, el pueblo es el titular del poder y hace ejercicio directo de él a través de su participación en los asuntos públicos, lo que da lugar a entender que se trata de un auténtico gobierno del pueblo. Aquella, por su parte, se instituye merced a la acción de unos representantes de la opinión y del interés de los ciudadanos en el marco de la ley, lo que hace que se constituya en "un sistema de limitación y control del poder, en el que cabe hablar, más que de un gobierno del pueblo, de un gobierno querido por el pueblo" (Cortina 2001, p. 89).

Crawford Macpherson elaboró una metodología para estudiar lo que llamó “los modelos democráticos" (Macpherson 1976). Allí nos presenta un modelo que llama "la democracia como participación". Analiza el problema que presentan las dimensiones de las sociedades modernas para aumentar la participación política y social. Muestra las dificultades que tendría llegar a una democracia participativa, dadas las características de la sociedad de mercado actual, la cual forma y requiere individuos orientados a la actividad económica productiva y de consumo, y desincentiva la preocupación por los problemas sociales y políticos considerados solo como el marco para la realización de los proyectos individuales (Vergara 1999).

Esta metodología fue continuada y desarrollada por David Held en su Models of Democracy (1987). Allí presenta un "modelo de democracia participativa", construido a partir del análisis de las teorías de Macpherson (2005), Pateman (1970) y Poulantzas (1978) (Vergara 1999). En su opinión, se requeriría una sociedad participativa que mejore la eficacia política, estimule la preocupación por los problemas colectivos y contribuya a formar "una ciudadanía sabia, capaz de interesarse en forma continuada por el proceso de gobierno" (ibíd). Sus características principales serían la participación directa de los ciudadanos en las instituciones clave del sistema político, en los lugares de trabajo y en la comunidad local; la reorganización del sistema de partidos para hacer participativos y responsables a los dirigentes frente a sus afiliados; la apertura del sistema institucional para mantenerlo abierto a la experimentación con nuevas formas políticas.

Por otra parte, los desarrollos en teoría política se han orientado durante las últimas décadas también hacia las teorías elitistas de la democracia. Los referentes teóricos predominantes en este sentido han sido Schumpeter (1942), Hayek (1944), la Public Choice Theory y otros. Esta tendencia a convertir a las élites políticas en las protagonistas del proceso democrático ha sido paralela a las dinámicas económicas y 
políticas de la globalización que tienden a la concentración del poder tanto nacional como internacional, político como económico. Ambas tendencias políticas y sociales se oponen al principio fundante de las democracias participativas que es el de la necesidad de la socialización del poder (Vergara 1988).

Las transformaciones de la teoría democrática podrían explicarse por varias razones. Se había ido produciendo un agotamiento de la teoría precedente de Laski, Dewey y otros, la cual era excesivamente normativa y carecía de descripciones realistas sobre la realidad democrática (Macpherson 1977). Su base empírica era escasa y su fundamentación provenía de la filosofía política. Asimismo, se basaba en el principio de la primacía de la política sobre la economía. Estos autores creían con excesivo optimismo o irrealismo que las estructuras económicas podían ser redefinidas o reguladas directamente mediante las decisiones políticas. Lo sucedido después de la Segunda Guerra fue más bien lo opuesto. Los sistemas políticos democráticos del Norte se hicieron más elitistas, se convirtieron en "tecnodemocracias", basadas en la articulación o integración de las elites político-tecnocráticas gubernativas y privadas, en la cuales la lógica económica fue la predominante (Duverger 1975). Este fenómeno, luego expandido universalmente en el mundo occidental, ha suscitado a partir del último tercio del siglo XX una de las discusiones más relevantes de la ética y la filosofía política contemporáneas.

De algún modo, esta expresión de la democracia que se acaba de describir, ha sido también enfrentada críticamente en la obra Democracia fuerte, de Benjamin Barber, quien allí objeta el concepto de "democracia blanda", que es la que, a su juicio, se origina cuando una sociedad se deja arrastrar irrestrictamente hacia el modelo de tipo decididamente liberal (Barber 2004), constituyéndose, entonces, la "democracia fuerte" en su antítesis. Barber supone que buena parte de los problemas de nuestro tiempo están ya implícitos en la propia teoría política que da sustento a la democracia liberal, es decir, sus fundamentos mismos no serían estrictamente democráticos. La primacía del interés individual que consagra la democracia liberal estaría en abierta oposición a la concepción más clásica de la democracia. Todo ello hace a Barber sostener que la teoría liberal sería la expresión más "blanda" de la democracia. Su particular fisonomía repercute, a su juicio, en una deflación ostensible de la participación ciudadana en la actividad política, con lo que la toma de decisiones concernientes a los bienes públicos queda remitida a la actividad de una particular clase de expertos profesionales, la clase de los políticos, que subrogan -en nombre de la ciudadanía- la titularidad de sus derechos a propósito de dichas decisiones (Barber 2004, pp. 394-395).

Sin olvidar las diferencias que Barber nos presenta respecto de los tres tipos o modalidades de democracia liberal (la del anarquismo liberal, la del realismo político y la del minimalismo político ${ }^{3}$ ), se puede colegir que el resultado siempre es el mismo:

Según Barber, la tendencia anarquista liberal se funda en la defensa exhaustiva del derecho individual, buscando que el poder político no intervenga ni lesione la acción de los individuos, salvo ante eventuales trasgresiones de la legislación. Por su parte, la tendencia del realismo político busca imponer la ley mediante el temor al castigo, considerándolo 
el ser humano de las democracias liberales, consumado en tanto "homo œconomicus"; egoísta, calculante, maximizador, inserto en la matriz de decisión racional para la que la democracia no constituye más que un instrumento en la búsqueda de su propio y aislado bienestar.

Por esta razón, la reflexión acerca de la democracia participativa parece indiscernible respecto de la reflexión acerca de la ciudadanía. Porque se entiende que los derechos de que los ciudadanos puedan gozar se constituyen en la fortaleza que les permite resistir la opresión y las limitaciones que las instituciones les pueden poner a su propio desarrollo. Es consustancial a la ciudadanía garantizar la dignidad humana. Por esto, se presume que un ejercicio efectivo de la ciudadanía generaría de tal manera un tipo de identidad entre ellos que les permitiría reconocerse y superar la desvinculación a que la modalidad predominante del desarrollo los induce (Cortina 2001). En la reflexión actual, la expansión de la concepción de ciudadanía, primariamente política, hacia sus facetas social, económica, civil, intercultural, favorece pensar en la rearticulación entre la razón sentiente individual y los valores y normas que tenemos por humanizadores, de modo que éstos puedan arraigar y ser asumidos por todos (ibíd).

\section{Hacia un concepto expandido e incluyente de "ciudadanía"}

Desde el afamado ensayo de T. H. Marshall, Ciudadanía y clase social (1950) ${ }^{4}$, la concepción más clásica acerca de la ciudadanía ha llegado a ser la suya. Como se sabe, en su ensayo, Marshall, conceptualiza la noción de ciudadanía según tres cuestiones fundamentales: la de los derechos civiles, la de los derechos políticos y la de los derechos sociales. Dicha conceptualización queda formulada de acuerdo con una periodización histórica particular, que permite entender, conjuntamente, el desarrollo de tipos particulares de derechos y el desarrollo histórico del período comprendido entre los siglos XVIII y XX. De este modo, el siglo XVIII queda asociado al surgimiento de los derechos civiles (libertades individuales, de palabra y conciencia, derechos de propiedad, de contratación y de igualdad ante la ley); el XIX al de los derechos políticos (participación en el ejercicio del poder político como elector o representante de los electores) y el XX al de los derechos sociales, respectivamente. Esta tercera etapa evolutiva que Marshall definió como la de la "ciudadanía social" y que dio forma al modelo de Estado de Bienestar, con diversos grados de ajuste y sometida a críticas de diversa índole, ha venido transitando históricamente desde entonces hacia la expectativa de una democracia social más completa e igualitaria.

el recurso estabilizador de la política. Finalmente, la tendencia del minimalismo político, busca implementar una suerte de término medio, o de equilibrio, entre el poder absoluto y la libertad absoluta (Barber 2004, pp. 350 y ss.).

$4 \quad$ Marshall, T. H. (1950). Citizenship and social class and other essays. Cambridge: Cambridge University Press. 
Los derechos sociales, destacados por Marshall, son concebidos como un tipo especial de derechos, puesto que no se obtienen de la pertenencia a una clase social ni expresan la existencia de alguna necesidad específica que los justifique, sino que son adquiridos por el simple hecho de que los individuos sean ciudadanos, es decir, estén en posesión de una "ciudadanía", que es aquello que equivale a ser titular de un conjunto de derechos y deberes, iguales para todos, codificados jurídicamente, los cuales deben ser obligatoriamente respetados y protegidos, y cuyo respeto y protección puede ser, a la vez, exigido (Andrenacci 2001, p. 2). Desde esta perspectiva, la noción de ciudadanía se funda en una perspectiva tanto universal como igualitaria. Lo que el estatuto de ciudadano garantiza a los individuos es que no operen sobre ellos procesos de exclusión o de discriminación, al menos de un modo básico y a modo de prevención de los fallos y desigualdades de corte material que los sistemas políticos y económicos contemporáneos tienden casi inevitablemente a generar (Cortina 2002a).

Para Marshall, "la ciudadanía, junto a otras fuerzas externas a ella, ha modificado el modelo de la desigualdad social" (Marshall 1998, p. 74). En su opinión, el enriquecimiento de que ha sido objeto el estatuto de la ciudadanía, a partir del siglo XX, "ha hecho más difícil conservar las desigualdades económicas, porque les deja menos espacio y aumenta las probabilidades de luchar contra ellas" (ibíd., p. 76). Y aunque hoy en día no se persigue la igualdad absoluta -porque la tendencia igualitaria admite límites, en un doble sentido, en parte a través de la ciudadanía, pero, en parte también, a través del sistema económico-, de lo que se trata es de eliminar las desigualdades consideradas ilegítimas (ibíd.). Se trata, por cierto, de contener y remontar la incapacidad que el sistema político y económico tiene para impedir las discriminaciones y otorgar igualdad social y económica e igualdad política, proveyendo iguales oportunidades a todos los individuos y favoreciendo su mejor participación en el sistema.

Por cierto, estas pretensiones de igualdad y de universalidad, puramente formales, contenidas en el espíritu del estatuto ciudadano, se ven limitadas en su materialización efectiva a la hora de articularse la práctica social de la individualidad y verse el ciudadano constreñido por el funcionamiento real del Estado y los diversos sistemas que lo constituyen (social, político, jurídico, económico). Lo que también podría ser entendido como un resultado de las prácticas de "gubernamentalidad" liberal descritas por Foucault en Seguridad, territorio, población (Foucault 2006, p. 137) -al menos en el segundo de los sentidos allí planteados.

El liberalismo democrático moderno ha permitido que se produzca una curiosa escisión entre aquello que consagra como igualdad de las personas ante la ley y aquello que establece como reglas de un juego económico que favorece significativas desigualdades individuales, al generar estratos de diferencia que tienden a constituirse en compartimientos estancos, lo que, en la práctica, niega la pretendida igualdad declarada jurídicamente. Ello redunda, como a menudo se entiende, en el establecimiento de un sistema de inequidades sociales y económicas que no muestran ser el correlato empírico de un sistema de derechos, pertenencias e igualdades. De tal manera, la noción de ciudadanía tiende a volverse equívoca e imprecisa, dado que el rasgo más definitorio por el que ha llegado a ser comprendida en la reflexión contemporánea -tras las diversas expresiones registradas en su evolución histórica-, el de la igualdad, tiende a perderse 
de vista cuando se ve fuertemente oscurecido por la diseminación de este factor de disparidad que los agenciamientos del Estado liberal democrático no pueden contener. La consolidación fáctica y progresiva de un régimen de desigualdades y exclusiones como el que transversalmente ha perfilado el desarrollo del mundo occidental a partir del siglo XVIII (habida cuenta del paréntesis más exitoso del Welfare state), desmiente el espíritu esencial del sentido que funda a la noción moderna de ciudadanía social.

Es el reconocimiento de esta tensión lo que ha movido a autores como Adela Cortina, por ejemplo, a enfatizar la distinción entre "bienestar" y "justicia" y a resaltar el diverso carácter que puede adoptar el Estado de derecho, en tanto exista como "Estado liberal de derecho" o como "Estado social de derecho", según lo que provea fundamentalmente sea bienestar o justicia (Cortina 2001, pp. 75-93). Cortina sostiene que "los mínimos de justicia que pretende defender el Estado social de derecho constituyen una exigencia ética", la que de ninguna manera puede quedar desatendida, como sí acontece en tanto lo que se enfatiza es principalmente el bienestar. El Estado social de derecho incluye y busca asegurar entre los derechos fundamentales, además de las clásicas libertades civiles y políticas, otros derechos sociales, económicos y culturales. Sería, entonces, la modalidad de Estado social de derecho la que cumpliría de modo efectivo la exigencia de justicia implicada en el carácter igualitarista y universalista de la noción de ciudadanía moderna, de esa noción canonizada de ciudadanía (social) a la que alude buena parte de la reflexión actual. "Mal podrá ejercer su libertad civil y su autonomía política quien carece de los recursos materiales básicos para hacerlo", nos recuerda Cortina, haciendo explícita la inconsistencia de fondo que afecta a dicho concepto de ciudadanía (ibíd., p. 91). La comunidad protege la autonomía de sus miembros, dice Cortina, cuando les reconoce derechos civiles y políticos; y aunque estos se consideren imprescindibles, no parece concluido su deber sino hasta cuando también los haga partícipes de los bienes sociales necesarios para que puedan tener una vida digna, bienes tan indispensables para una vida auténticamente humana que no pueden quedar sometidos a los vaivenes del mercado (ibíd., p. 93).

Ahora bien, concebir la ciudadanía en estos términos equivale a atribuir un protagonismo más bien secundario a los sujetos portadores del estatuto ciudadano, a concebirlos como estando simplemente "sujetos" a esa provisión de derechos que reciben sin que medie su propia agencia. Una constatación de este tipo ha conducido a la teoría respectiva a proponer la corrección de semejante deficiencia implicada en el concepto canónico de ciudadanía social que fuera teorizado inicialmente por H. T. Marshall, en el sentido de una ampliación conceptual que permita pensar en un concepto de ciudadanía "activa", que dé cuenta de una disposición comprometida y responsable con sus propias posibilidades por parte de los mismos ciudadanos.

Esta necesidad de expansión del principio de la ciudadanía y la corrección de sus eventuales insuficiencias ha implicado el reconocimiento de otras dimensiones constitutivas suyas, sin las cuales parece imposible una comprensión más cabal de su sentido. Entre otras, la faceta económica de la ciudadanía se ha convertido en el objeto de la reflexión. Por cierto, las preocupaciones actuales por una "ciudadanía económica" se vinculan a la idea de que en la esfera de la actividad económica "los afectados por las decisiones que en ella se toman son sus propios señores" y no súbditos; lo cual implica 
en buena ley que han de participar de forma significativa en la toma de decisiones que les afectan" (ibíd., p. 99). La ampliación de la noción de ciudadanía hacia el reconocimiento de una ciudadanía económica tendría necesariamente que plantearse, en este caso, una resignificación contundente de lo económico; tendría que contribuir a poner un freno de importancia a la actual tendencia privatizadora del sistema global de la economía y contener la concentración extrema de la riqueza, así como el usufructo desigual de los bienes materiales que caracteriza al mundo actual. Esta desigualdad da lugar a sociedades cada vez más fracturadas y excluyentes, sustentadas en una creciente concentración de poblaciones alienadas de la economía, imposibilitadas de ejercer sus derechos en materias económicas, excluidas del todo de las decisiones de tal naturaleza que definen su propia existencia, y que estando privadas de la posibilidad de elegir la vida respecto de la cual tienen razones para considerarla valiosa (Sen 1998a) deben contemplar con resignación la opulencia insolente con que perfila la suya un sector minoritario de la población. En este momento histórico de transición hacia las sociedades tecnológicas avanzadas la noción de ciudadanía económica debe cumplir un papel semejante al que cumpliera hasta ahora la noción de ciudadanía social (Tezanos 2004).

Otra dimensión que en este giro expansivo de la reflexión contemporánea acerca de la ciudadanía ha cobrado interés entre diversos teóricos es, sin duda, aquél que refiere a las severas dificultades que se ponen de manifiesto en el mundo actual en relación con la convivencia entre individuos representantes de denominaciones culturales diversas; un fenómeno derivado del hecho de que casi todos los países del orbe son prácticamente multiétnicos hoy en día. Lo que a su vez lleva frecuentemente a que se establezca "una distinción entre «cultura de primera» $\mathrm{y}$ «culturas de segunda» que suscita sin remedio sentimiento de injusticia y desinterés por las tareas colectivas" (Cortina 2001, p. 178). En este sentido, Will Kymlicka, aludiendo al hecho de que acontecimientos como la apatía política de los electores o la tensión centroeuropea originada a partir del incremento de la población multicultural y multirracial, o el fracaso de las políticas ambientales basadas en la cooperación voluntaria de los ciudadanos (Kymlicka 1996, p. 241), deja en claro que la estabilidad de las democracias modernas no depende únicamente de la justicia de sus instituciones básicas, sino también de las cualidades y de las actitudes de sus ciudadanos; vale decir, del sentimiento de identidad que éstos sean capaces de producir, a la vez que de las apreciaciones que lleguen a tener de "otras formas de identidad nacional, regional, étnica o religiosa que potencialmente pueden competir con la suya; de su capacidad de tolerar y de trabajar con personas distintas de ellos" (ibíd.). Ello es un componente de importancia a la hora de evaluar su deseo de asumir un compromiso ciudadano, de decidirse a participar en el proceso político tendiente al establecimiento del bien público, de reconocer y fortalecer los vínculos con las autoridades responsables y de asumir de manera personal un sentido de justicia que favorezca una distribución más equitativa de los recursos.

Parece ser cierto, siguiendo a Kymlicka, que sin ciudadanos capaces de enfrentar estos desafíos, las sociedades, sobre todo aquellas multinacionales o pluriétnicas, ven mermado su potencial de crecimiento y desarrollo, porque "los mecanismos procedimentales e institucionales no bastan para equilibrar los intereses de cada uno, [...] [por lo que] es necesario cierto grado de virtud cívica y de espíritu público" (ibíd., 
p. 242). A Kymlicka le parece claro que si existe una manera viable de promover el sentimiento de solidaridad y hacer converger en una perspectiva común las metas de las personas en los Estados multinacionales, ello es solo mediante la acomodación de la diversidad de identidades y nunca a partir del sometimiento de algunas de ellas. Afirma en tal sentido: "Las personas de diferentes grupos nacionales únicamente compartirán una lealtad hacia el gobierno general si lo ven como el contexto en que se alimenta su identidad nacional y no como el contexto que la subordina" (ibíd., p. 259). Una perspectiva de ciudadanía multicultural, luego, concerniría a la defensa del derecho a reivindicar los valores poliétnicos de los grupos de inmigrantes y de otros sujetos sociales desfavorecidos, con miras a su eventual inclusión en el juego social, de manera que puedan adquirir una participación plena, asunto que, como hemos señalado, no depende exclusivamente del marco institucional ni del otorgamiento de los derechos sociales en los que pensó Marshall.

Reiterando, Kymlicka supone que todos estos problemas asociados a una "política de la diferencia", que es vista por muchos como una verdadera amenaza a la democracia liberal, pueda ser "gestionada" de una manera pacífica y a la vez justa, asumiendo, sí, que exista un cierto grado de buena voluntad de parte de los ciudadanos. No deja de reconocer por ello, que en diversos lugares los diferentes grupos están menos motivados por la buena voluntad que por el odio y la intolerancia y que existen altas probabilidades de que los grupos étnicos abusen de sus derechos y poderes. Un concepto afinado de ciudadanía multicultural tendría, deducimos, que establecer al menos dos restricciones a esta posibilidad. Por una parte, limitar el hecho de que el otorgamiento de derechos desemboque en la dominación de un grupo sobre otro u otros, y, en segundo término, que al interior de los propios grupos haya unos miembros que dominen a otros de sus iguales. En otras palabras, se debería tratar de "asegurar que exista igualdad entre los grupos, así como libertad e igualdad dentro de los grupos” (ibíd., p. 266).

\section{Una ciudadanía biológica}

Llama la atención una dimensión reciente que en la reflexión contemporánea se ha comenzado a escrutar en relación con el asunto de la ciudadanía, en vistas de su ampliación teórica. Se trata del asunto de la "ciudadanía biológica". Pero, deberemos aclarar algunas cuestiones previas para su mejor comprensión.

Desde una perspectiva crítica, el teórico francés Didier Fassin examina la obra de Foucault para detectar que su reflexión sobre la biopolítica, iniciada en el año 1976 con el volumen I de la Historia de la sexualidad ("La voluntad de saber") (HS1), constituye un trabajo inconcluso, que, aunque nunca abandonó de manera decidida, tampoco lo retomó luego del curso en el Collège de France de 1979 Nacimiento de la biopolítica (Fassin 2010, p. 22). Curiosamente, y a diferencia de lo que se proponía en su título (algo ya muy comentado, por lo demás), el curso de aquel año estuvo dedicado enteramente al estudio del liberalismo. Fassin recuerda al respecto que, meses después, con ocasión de la redacción de la síntesis anual preparada para los Anales del Collège, Foucault 
señaló que la tarea siguiente que correspondía acometer era el estudio del modo cómo los problemas específicos de la vida y la población se habían presentado en el contexto de una tecnología de gobierno definida desde el siglo XVIII por el liberalismo. Aunque ello, en verdad, jamás ocurrió. Los "problemas específicos de la vida" no volvieron a figurar en los cursos posteriores dictados hasta antes de su desaparición en 1984. Otro tanto, a juicio de Fassin, habría acontecido con el asunto del biopoder, tema enunciado primariamente en la obra del 76 y luego brevemente aludido en el comienzo del curso de 1978, Seguridad, territorio, Población (ibíd.), pero nunca abordado directamente, conforme al ofrecimiento hecho por Foucault en este último trabajo. Fiel a su inspiración nietzscheana -supone Fassin- Foucault habría descuidado las cuestiones de la vida en sí, por razones de método, que lo llevaron a sospechar del significado y a abandonarlo. "Foucault cree - dice el comentarista- que no existe un significado secreto o profundo y que la interpretación debe permanecer al nivel superficial de las cosas" (ibíd., p. 24). La vida no habría sido su principal preocupación, piensa, "ni como bios ni como zoé". Foucault habría estado interesado solo en el modo en que unos seres vivientes impersonales eran convertidos en poblaciones o en individuos; es decir, en la manera en que la gubernamentalidad y los procesos de subjetivación llegaron a constituir la moderna visión del mundo y de la humanidad (ibíd., p. 25).

Ello daría lugar, a juicio de Roberto Esposito, a una "biopolítica negativa" (biopoder o biocracia) que sería "la que se relaciona con la vida desde el exterior, de manera trascendente, tomando posesión de ella, ejerciendo la violencia. Como ocurrió de la manera más catastrófica con el nazismo y sigue ocurriendo hoy en muchas partes del mundo". Esta biopolítica negativa se relaciona con la vida a través de la muerte; opera despojando a la vida de su carácter formal, de su calificación, y la reduce a simple materia viviente (zoé). Fragmenta a la vida (bíos) en regiones de diferente valor. Subordina a aquellas consideradas o sin valor, o de escaso valor, y privilegia a las que se les atribuye mayor valor biológico. "El resultado de este procedimiento es una normalización violenta que excluye lo que se define preventivamente como anormal y, al fin, la singularidad misma del ser viviente" (Castro 2005).

Sin embargo, Didier Fassin reconoce una excepción en cuanto a esto. Tanto al final del curso del año 1976, Defender la sociedad (DS), como en el último capítulo de HS1 se encontraría una diferencia. DS evocaría el giro desde el derecho soberano de "hacer morir y dejar vivir" hacia el biopoder de "hacer vivir y dejar morir", de un modo semejante a lo propuesto en HS1, cuando Foucault distingue la noción aristotélica de hombre como animal viviente y existencia política, de la noción moderna de hombre como animal en cuya política está puesto en entredicho su ser viviente (Rose 2001, p. 1). Fassin piensa que tales aseveraciones muestran que lo que aquí está en cuestión es la vida y la muerte y no las conductas. Vale decir, en vez de interesarse por las tecnologías de poder, Foucault se habría interesado acá en el objeto del poder, en ese "poder sobre la vida", que señala en HS1. Tanto el contenido de esta reflexión como su tono trágico (Foucault alude al exterminio racista estatal del nazismo) no se repetirían posteriormente en su trabajo, consagrado por entero al asunto de la gubernamentalidad y la subjetivación. 
Vistas así las cosas (y aceptado este necesario circunloquio), Fassin busca seguir los pasos perdidos de Foucault para desarrollar algunas "implicancias del concepto de biopolítica" -aquellas previstas pero abandonadas por el filósofo en su obra tardía-, abordando la cuestión de lo que, en coincidencia con Nikolas Rose, llama "la vida en sí": "la vida que es vivida a través de un cuerpo (no solo por medio de las células) y como sociedad (no solo como especie)" (Rose 2006, p. 27). Tal concepción busca escapar a la restricción habitual que remite la vida a simple fenómeno biológico (aunque también lo sea) y al hecho de que los seres vivientes sean reducidos a poblaciones (aunque sí lo son desde una perspectiva estatal). Busca, en definitiva, regresar "al punto al que llegó Foucault antes de limitar la biopolítica a sus tecnologías y la moral a la ética" (ibíd., p. 29). Este trabajo constituye, de hecho, una reformulación de las tesis foucaultianas, en la que resaltan algunas cuestiones teóricas de interés para este artículo.

Entre otras cosas, afirma Fassin -con un espíritu correctivo respecto del texto de Foucault-, que las sociedades contemporáneas se caracterizan menos por la emergencia del biopoder que por la imposición de la "biolegitimidad", más por el poder de la vida misma que por el poder sobre la vida, como señalaba el filósofo. Esto último correspondería a aquello que algunos autores, como Adrian Petryna por ejemplo, han llamado ciudadanía biológica (Petryna 2000) y constituiría una pieza clave en la reformulación de la reflexión foucaultiana. Asumiendo etimológicamente el término, la biopolítica se presenta no tan solo como política de la población, sino como política de la vida y tiene que ver, en especial, con las desigualdades que la afectan, a las que Fassin llama "biodesigualdades". En este sentido, de lo que se trataría no es únicamente de la "normalización" de la vida de las personas -según el conocido precepto- sino más bien de la decisión respecto del tipo de vida que éstas pueden o no vivir. Ello hace que el pensamiento sobre la biolegitimidad y las biodesigualdades cobre una relevancia fundamental a la hora de atribuir significado y valor, tanto a "la vida en sí", como a las vidas concretas de los individuos, por lo que se podría entender que "la biolegitimidad se ha convertido en una cuestión central en las economías morales de las sociedades contemporáneas" (Fassin 2009, p. 32) y, por lo mismo, en un asunto clave para su definición política profunda. Una "razón humanitaria" constituye el sello distintivo de la política actual, según afirma el crítico. Ésta se habría convertido en un modo generalizado de acción para el gobierno de los hombres. Ejemplos como los de las políticas francesas de inmigración, que a partir de la década de los 90, conjuntamente con restringir drásticamente el asilo por causas políticas, han permitido un incremento significativo de la legalización de indocumentados portadores de enfermedades; o el caso de la asignación de un estatuto político diferencial que el gobierno ruso otorgó a la víctimas del desastre de Chernobyl, por el cual pudieron acceder a compensaciones y beneficios extraordinarios, revelan el cambio en las políticas de la vida que han venido teniendo lugar en el mundo durante las últimas décadas. "Se prefiere la vida del enfermo, con su padecimiento actual y su evidencia física, a la del refugiado, con su pasado doloroso y su significado político" (ibíd., p. 34).

No se trataría, en consecuencia, de una política mediante la cual la ley, sea la ley moral o la jurídica, es impuesta sobre los cuerpos en nombre, por ejemplo, de prescripciones de sanidad, para evitar enfermedades (sería el caso de las políticas de 
salud pública), o de prescripciones de normalidad (sería el caso de la contención de las desviaciones respecto del orden público). Por el contrario, según Fassin, en tal sentido se trataría de "una política en la cual es el cuerpo el que da derecho, a título de la enfermedad (justificación de atención médica) o del sufrimiento (apelando a la compasión), ya sea a una tarjeta de residencia, ya sea a una ayuda financiera de emergencia" (Fassin 2003). El relato acerca del sí mismo en vistas de la obtención de ayuda social, o la exposición del padecimiento para regularizar por motivos médicos una condición ilegal de extranjería o bien mostrar las secuelas de padecimiento producido por la tortura física, o la narración directa de la condición vital deprivada para obtener amparo, y otras semejantes, son todas expresiones contundentes y frecuentes del avance de la bio-legitimidad, puesta en práctica cada vez más en la escena internacional por una razón humanitaria frente a todos los conflictos. La reivindicación de esta "ciudadanía biológica", articulada a través del reclamo contenido en la narración del sí mismo, constituye una nueva figura en el gobierno contemporáneo de los hombres, en este caso particular, en el gobierno de los dominados (ibíd.).

Desde comienzos del siglo presente, al que muchos han calificado como el "siglo biotecnológico" (Rose 2006), cobra particular relevancia esta ampliación de la noción de ciudadanía hacia su dimensión biológica, porque muestra inequívocamente un potencial correctivo respecto de la concepción tradicional con la que Marshall formuló inicialmente el problema, definiéndolo como asignación de derechos sociales que buscaban consagrar una perspectiva igualitarista, la misma que las democracias liberales hasta ahora no han podido sostener, habiendo establecido, más bien, un régimen de inequidades oprobiosas que han conseguido que los derechos tengan un carácter puramente nominal. Entender la política más allá de la gubernamentalidad, por tanto, equivale también a poner atención sobre el trato que se otorga a las personas y el modo en que sus vidas resultan ser evaluadas "más que sobre las tecnologías [de todo tipo] que operan en estos procesos" (ibíd.). Porque lo que la política es capaz de hacer con la vida de las personas no remite únicamente a un asunto de discursos y tecnologías -cree Fassin-, o de estrategias y tácticas. Tiene que ver con el modo real en que los grupos y los individuos son tratados, según qué moral o qué principios se les evalúa, conforme a qué tipo de desigualdades y en ausencia de qué reconocimientos se les excluye (ibíd., p. 45). Esclarecedoras resultan al respecto las siguientes palabras de Esposito: "En general, una biopolítica afirmativa es la que establece una relación productiva entre el poder y los sujetos. La que, en lugar de someter y objetivar al sujeto, busca su expansión y su potenciación. [...] Naturalmente, para que el poder pueda producir, en vez de destruir la subjetividad tiene que serle inmanente, no tiene que trascenderla. Así, la norma no tiene que gobernar o discriminar a los sujetos desde lo alto de su generalidad, sino que tiene que ser absolutamente singular como cada vida individual a la que se refiere. Se podría, en fin, hablar de política de la vida y no sobre la vida. No solo si la vida, cada vida individual, es sujeto y no objeto de la política, sino también si la misma política es repensada mediante un concepto de vida de acuerdo con toda su extraordinaria complejidad interna, sin reducirla a la simple materia biológica" (Castro 2005).

Por ello, no es irrelevante que se adopte una u otra decisión en materias de salud pública o de políticas sociales, en los temas del empleo, la vivienda o la educación. 
Todo esto impacta de manera determinante en la expectativa de vida de los sujetos y las poblaciones, decide la duración promedio de sus vidas. En consecuencia, una noción de derechos ciudadanos, ampliada hasta incluir la dimensión biológica de la existencia de las personas, es decir, una "ciudadanía biológica", resulta ser clave a la hora de pensar en la consolidación de aquella igualdad prometida por la concepción original de la ciudadanía social.

\section{Conclusiones}

En este artículo se ha perseguido el propósito de establecer una vinculación teórica y productiva entre las nociones de Ética del Desarrollo, democracia participativa (deliberativa) y ciudadanía biológica. Para ello se ha tenido en cuenta, en primer lugar y al modo de un insumo conceptual, el corpus desplegado por un conjunto de pensadores actuales que, pese a ciertas notas distintivas que caracterizan sus respectivos trabajos, han avanzado de manera común sobre una idea peculiar con respecto al fenómeno del desarrollo económico, dando lugar a una reflexión crítica sobre los fines y medios que definen los cambios sociales, económicos y culturales en los países y regiones pobres y en aquellos otros así llamados "en vías de desarrollo". Como se señaló en un comienzo, el rasgo distintivo de su reflexión busca destacar el papel activo que le cabe jugar a la dimensión ético-moral de la teoría y la práctica del desarrollo, paralelamente a sus componentes económicos, políticos y tecnocientíficos. El presupuesto de base es que no resulta legítima una visión puramente economicista del desarrollo, porque éste no puede ser comprendido solo en relación con el crecimiento del PIB o el aumento de indicadores de la renta nacional, como suele ser presentado por la lógica económica prevaleciente en la actualidad. Parece necesario abordar las causas de la desigualdad económica global, el hambre y el subdesarrollo y la serie de otros problemas que a estos fenómenos se vincula, con una concepción del desarrollo explícitamente basada en principios éticos. El desarrollo no debe ser medido sin tener en cuenta el estilo de vida que pueden llevar las personas y sus libertades reales. El objetivo fundamental del desarrollo debe ser, entonces, dotar a las personas de las "capacidades" necesarias para que puedan alcanzar las realizaciones que les reportan satisfacción y puedan vivir el tipo de vida respecto de la cual tienen razones para considerarla valiosa, es decir, se trata de que las personas actúen, desplieguen su agencia y generen cambios y materialicen logros que puedan ser juzgados en función de sus propios valores y objetivos, independientemente de que puedan ser evaluados o no, además, en función de otros criterios externos a ellas mismas.

No obstante -y aquí se visualiza el primer gozne de la articulación pretendida por este artículo-, un empoderamiento tal de los sujetos solo resulta concebible en el contexto de una transformación sustancial del esquema histórico de desarrollo y funcionamiento del sistema democrático, consolidado en nuestros días bajo una fisonomía puramente representativa, devenido inconsistente e insatisfactorio para tales fines. En vistas de ello, se requeriría una estructura democrática participativa 
(deliberativa), que aumentara la eficacia política, estimulara la preocupación por los problemas colectivos y contribuyera a formar una ciudadanía capaz de interesarse de manera continuada por el proceso de gobierno. Una democracia participativa debería permitir la participación directa de los ciudadanos en las instituciones clave del sistema político, en los lugares de trabajo y en la comunidad local; tendría que acometer una reorganización del sistema de partidos políticos para hacer aumentar las cuotas de responsabilidad dirigencial y abrirse a nuevas formas políticas, cuando menos. La democracia participativa (deliberativa), de acuerdo a este modelo, requeriría de ciertas condiciones generales de posibilidad: aumentar los recursos materiales de los grupos sociales; disminuir el poder burocrático no responsable ante los ciudadanos; mantener un sistema abierto de información que posibilite decisiones informadas; pues, lo que define al concepto de democracia participativa es la deliberación en sí misma. Cuando los ciudadanos deliberan, intercambian puntos de vista y debaten sobre asuntos de políticas públicas, suponen que su opinión política debería ser examinada con otros ciudadanos; es en este punto en el que la razón publica es de crucial importancia, dado que caracteriza el razonamiento de los ciudadanos sobre elementos constitucionales esenciales y asuntos de justicia básica.

Se trata, como algunos lo han sostenido, de uno de los retos más decisivos para nuestro tiempo, porque requiere de un robustecimiento y una expansión de la noción clásica de ciudadanía -y he aquí el segundo gozne de la articulación propuesta en este artículo, el que cierra esta argumentación. Dada la promesa igualitaria incumplida de las democracias modernas (liberales), por la abdicación que el Estado ha hecho de su papel regulador, las condiciones de vida que alguna vez garantizó, o tuvo en mente garantizar, por medio de la regulación ejercida sobre el mercado y los salarios, se han visto severamente mermadas. Las desigualdades sociales y económicas generadas como consecuencia comprometen el estatuto de la ciudadanía porque se traducen no solo en la pérdida de los derechos, a la asistencia social y a la provisión estatal de los servicios, sino en la eliminación efectiva de los derechos de participación en los asuntos públicos, porque directa o indirectamente, las carencias socioeconómicas desembocan en exclusión política y en la pérdida de legítimos reconocimientos. La literatura filosófico-política contemporánea muestra una proliferación de alcances teóricos al respecto. Tal como anteriormente se ha mostrado, al concepto canónico de ciudadanía levantado inicialmente por Marshall, se le han agregado reflexiones que buscan ampliar su definición, a través de la incorporación de algunas dimensiones no tematizadas anteriormente como parte esencial de su significado De tal modo, los conceptos de "ciudadanía económica" o de "ciudadanía multicultural" - por mencionar algunos, ya que hay otros-imponen notas diferenciales que no solo revitalizan el adelgazado carácter de la clásica "ciudadanía social", sino que, a la vez, perfilan elementos de debate filosófico centrales para un mejor escrutinio del presente.

Siguiendo este razonamiento, este artículo propone mirar con particular interés, a modo de corolario, el concepto de "ciudadanía biológica", porque parece representar una apertura significativa del tema para futuras contribuciones y a la vez cerrar esta reflexión en sintonía con ciertas preocupaciones y problemas propios del presente. A través del examen de la categoría de "ciudadanía biológica", la matriz analítica de la biopolítica 
foucaultiana -a estas alturas convertida en una verdadera koiné de nuestros días-puede verse reconsiderada en términos de una "biopolítica afirmativa", en consonancia con la realidad de un siglo biotecnológico por excelencia, donde las preocupaciones por una biolegitimidad no pueden ser menores ni intrascendentes.

En síntesis, un buen desarrollo, un desarrollo entendido en clave ética, necesita de una estructura de democracia participativa, que permita la deliberación activa y comprometida de unos ciudadanos diversos y empoderados, que reivindiquen sus derechos teniendo en vista, en último término, la vida en sí misma.

\section{Referencias bibliográficas}

Andrenacci, L. (2001), De civitas Inaequalis. Elementos para una teoría de la ciudadanía, en www.saap.org.ar/esp/docs-congresos/.../V/.../luciano-andrenacci. pdf (consultado el 10.08.12)

Bachrach, P. (1967), Crítica a la teoría elitista de la democracia. Buenos Aires: Ed. Amorrortu.

Barber, B. (2004), Democracia fuerte. Córdoba: Almuzara.

Castro, E. (2005), "Toda filosofía en sí es política. Entrevista a Roberto Esposito", Revista N, Diario Clarín, Buenos Aires, edición del 12 de marzo de 2005.

Conill, J. (2006), Horizontes de economía ética. Madrid: Tecnos. Bancaja.

(coord.) (2002), Glosario para una sociedad intercultural. Valencia:

Cortina, A. (2000), Ética de la empresa. Madrid: Trotta.

(2001), Ética aplicada y Democracia radical. Madrid: Tecnos.

(2002a), Por una ética del consumo. La ciudadanía del consumidor en un mundo global. Madrid: Taurus.

(2002b), "El estatuto de la ética aplicada. Hermenéutica crítica de las actividades humanas", en Graciela Fernández, comp., El giro aplicado. Transformaciones del saber en la filosofía contemporánea. Ediciones de la Universidad Nacional de Lanús, Buenos Aires, pp. 61 a 77.

(2003), Ciudadanos del mundo. Hacia una teoría de la ciudadanía. Madrid: Alianza.

(2007), Ética de la razón cordial. Educar en la ciudadanía en el siglo XXI. Oviedo: Nobel.

(2009), Justicia cordial. Madrid: Trotta.

Cortina, A. y Pereira G., ed. (2009), Pobreza y Libertad. Erradicar la pobreza desde el enfoque de Amartya Sen. Madrid: Tecnos.

Crocker, D. (2002), "Ética internacional del Desarrollo: Fuentes, acuerdos, controversias, y agenda", en Joseph Stiglitz, comp., Ética y Desarrollo: La Relación Marginada. Buenos Aires: El Ateneo. 
(2004), Cross-cultural communication and International Development Ethics, manuscrito.

(2005), Sen and Deliberative Democracy, Capabilities equality: Issues and problems. London \& New York: Routledge.

(2008), Ethics of global development: Agency, capability, and deliberative democracy. Cambridge: Cambridge University Press.

Duverger, M. (1975), Las dos caras de Occidente. Barcelona: Ed. Ariel.

Elster, J. (1998), La democracia deliberativa. Barcelona: Gedisa.

Fassin, D. (2003), Gobernar por los cuerpos, políticas de reconocimiento hacia los pobres y los inmigrantes, Cuadernos de Antropología Social, № 17, Facultad Filosofía y Letras, Universidad de Buenos Aires.

(2007), Humanitarianism as politics of life, Public culture 19, 3: 499520.

(2009), Another politics of life is possible, Theory, Culture and Society, 26 (5): 44-60. Versión castellana en Lemm, V., editora, (2010). Neoliberalismo y Biopolítica. Ediciones UDP, Santiago (trad. D. Rosello).

Fishkin, J. y Laslett, P. (2003), Debating deliberative democracy. Oxford: Blackwell Publishing.

Foucault, M. (2006), Seguridad, territorio, población. Buenos Aires: F. C. E.

Goulet, D. (1999), Ética del Desarrollo. Guía teórica y práctica. Madrid: IEPALA.

Gutmann, A. y Thomson, D. (2004), Why deliberative democracy? Princeton N.J: Princeton University Press.

Habermas, J. (1998), Facticidad y validez. Madrid: Trotta.

(1999), La inclusión del otro. Barcelona: Paidós.

(2000), La constelación postnacional. Madrid: Paidós.

Hayek, F. (1993), Caminos de servidumbre. Madrid: Alianza.

Held, D. (1997), La democracia y el orden global. Del Estado moderno al gobierno cosmopolita. Barcelona: Paidós.

(2007), Modelos de democracia. Madrid: Alianza.

Höffe, O. (2007), Ciudadano económico, ciudadano del Estado, ciudadano del mundo. Ética política en la era de la globalización. Buenos Aires: Katz.

Kymlicka, W. (1996), Ciudadanía multicultural. Una teoría liberal de los derechos de las minorías. Barcelona: Paidós.

(2003), La política vernácula. Nacionalismo, multiculturalismo y ciudadanía. Barcelona: Paidós.

(2004), Estados, naciones y culturas. Madrid: Almuzara.

Kymlicka, W. y Norman, W. (2000), Citizens in diverse societies. UK.: Oxford University Press.

Macpherson, C. B. (2005), La teoría política del individualismo posesivo. De Hobbes a Locke. Madrid: Trotta. 
(2009), La democracia liberal y su época. Madrid: Alianza.

Marshall, T. H. y Bottomore, T. (1998), Ciudadanía y clase social. Madrid: Alianza.

Martínez Navarro, E. (2007), "Ética del desarrollo de los pueblos. Panorámica y perspectivas", Diálogo filosófico, año 23, septiembre/diciembre, III/07.

Nino, C. S. (2005), Constitución de la Democracia deliberativa. Barcelona: Gedisa.

Pateman, C. (1970), Participation and Democratic Theory, Cambridge University Press.

Petryna, A. (2000). Life exposed. Biological citizenship after Chernobyl. Princeton: Princeton University Press.

(2002), Life exposed. Biological citizenship after Chernobyl. Princeton: Princeton University Press (citado por Fassin 2009).

Peñas, P. (2011), Barber y la idea de Democracia fuerte. Revista Tales 4: 279-290.

Rose, N. (2001), “The politics of life itself”, Theory, Culture and Society 18 (6): 1-30.

Rostow, W. (1960), The stages of economic growth. A non-communist Manifesto. Cambridge University Press.

Schultz, T. (1959). Investment in man. An economist's view, Social Service Review 33: $110-117$.

Sen, A. (1998a), Sobre ética y economía. Madrid: Alianza. (1998b), Bienestar, justicia y Mercado. Barcelona: Paidós. (2000), Desarrollo y Libertad. Barcelona: Planeta. (2002), Nuevo examen de la desigualdad. Madrid: Alianza. (2005), Human Rights and Capabilities. Journal of Human Development 6 (2): 151-66.

Tezanos, J. F. (2004), Tendencias en desigualdad y exclusión social. Madrid: Sistema. Van Parijs, P. (1998), Libertad real para todos. Barcelona: Paidós.

Van Parijs, P. y Arnsperger, C. (2002), Éthique économique et sociale. Paris: La Decouvérte.

Vergara, J. (1999), La concepción de Democracia participativa de Habermas, Revista Ciencia política, Volumen XX, $\mathrm{N}^{\mathrm{o}} 1$.

(1988), Modelos elitarios de Democracia. Dianoia 1988. México D. F.: Instituto de Investigaciones Filosóficas de la Universidad Nacional de México y FCE.

Villarroel, R. (2009), Ética, Economía y Pobreza. Una posible articulación en clave hermenéutica, Anales del Instituto de Chile. Vol. XXVIII, Estudios "La pobreza en Chile" III. 